\title{
VOLUME CALIBRATION USING A COMPARISON METHOD WITH A TRANSFER LEAK FLOW RATE
}

\author{
F. Boineau ${ }^{1}$, M. D. Plimmer ${ }^{1}$, E. Mahé ${ }^{1}$ \\ ${ }^{1}$ Laboratoire commun de métrologie du LNE-Cnam, 1 rue Gaston Boissier, Paris, France, frederic.boineau@lne.fr
}

\begin{abstract}
:
This paper describes a volume comparison method carried out under a fine/rough vacuum using a small transfer flow rate from a capillary leak artefact. This method is suitable for volumes of vessels with complex shapes such as tubing arrangements equipped with various pneumatic parts (valves, gauges...), sample volumes, etc. The calibration, based on pressure rise measurements performed with a constant-volume flowmeter set-up, exhibits a standard relative uncertainty between $0.03 \%$ and $2 \%$ for volumes ranging from 1 to $3000 \mathrm{~cm}^{3}$, which is only about one order of magnitude higher than capabilities of the gravimetric volume calibration.
\end{abstract}

Keywords: Volume calibration; Vacuum; Constant-volume flowmeter; Pressure rise measurements; Capillary leak rate

\section{INTRODUCTION}

The most accurate way to determine the volume contained by a vessel is the so-called "gravimetric volume calibration" method [1], which consists in weighing the vessel, empty and then filled with a liquid (usually water) and calculating the volume from the mass and density of the liquid added. As long as the vessel is transparent or can be filled with confidence so that no air bubbles remain inside, this primary method can provide a relative standard uncertainty in the $10^{-5}$ range.

The comparison method presented here is suited to volumes either incompatible with liquids or else with internal shapes such that prevent complete filling. It is derived from the pressure rise method implemented in the Laboratoire commun de métrologie (LCM LNE-Cnam) constant-volume flowmeter [2] used to calibrate leak rates in the range from $1 \times 10^{-7}$ to $1 \times 10^{-2} \mathrm{~Pa} \cdot \mathrm{m}^{3} \cdot \mathrm{s}^{-1}$. The leak rate is deduced from the measured pressure rise rate and the total volume into which the gas flows. In this flowmeter, a standard volume, calibrated using the gravimetric method, is connected in series with the internal volume of the tubing which links the leak artefact to the pressure sensor, called the dead volume. This latter may be determined prior to the leak rate measurement. Setina and Erjavec have studied a similar arrangement to calibrate the volume of chambers in a set-up under high vacuum [3].

In the present work, the standard and unknown volumes are connected in parallel. The volume calibration using a comparison method with a transfer leak flow rate was originally developed for the needs of companies owning sample volumes in which some gas is trapped for a further analysis. As this kind of artefact is sometimes incompatible with the gravimetric volume calibration and has to be functionally tight to a certain extent, the comparison method is well suited.

\section{PRINCIPLE}

The principle is developed first for the arrangement where the standard and unknown volumes are connected in parallel. The necessary determination of the dead volume, corresponding to the connection in series of this dead volume and the standard volume, is presented on the same basis.

\subsection{Volumes connected in parallel}

A steady gas throughput $q$ from a capillary leak artefact is used. In a given volume $V$, at a constant temperature $T$, the pressure rise rate $\Delta p / \Delta t$ produced by the leak rate $q$ is such that:

$$
q(T)=V \frac{\Delta p}{\Delta t} \text {. }
$$

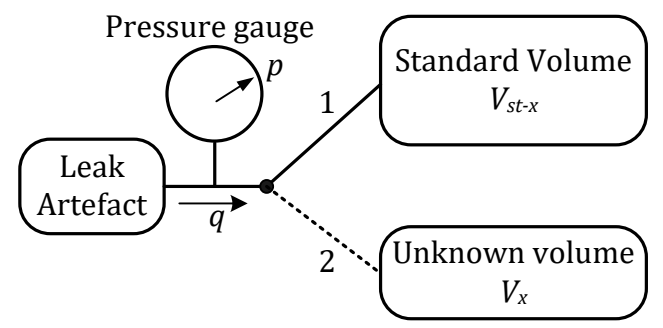

Figure 1: Calibration set-up, volumes connected in parallel.

The comparison method consists in directing the gas flow successively into a standard volume $V_{s t-x}$ 
(phase 1) and an unknown one called $V_{x}$ (phase 2), placed in a parallel arrangement (Figure 1).

The pressure is recorded over time during these phases, between two thresholds $p_{\min }$ and $p_{\max }$ (Figure 2). It takes times $\Delta t_{s t}$ and $\Delta t_{x}$ for the pressure to rise from $p_{\min }$ to $p_{\max }$ in volumes $V_{s t}$ and $V_{x}$ respectively. Assuming a constant temperature and no distortion of the walls of the volumes during measurements, the equality of $q(T)$ in $V_{s t}$ then $V_{x}$ leads via equation (1) to:

$$
V_{s t-x}\left[\frac{p_{\max }-p_{\min }}{\Delta t_{s t}}\right]=V_{x}\left[\frac{p_{\max }-p_{\min }}{\Delta t_{x}}\right] \text {, }
$$

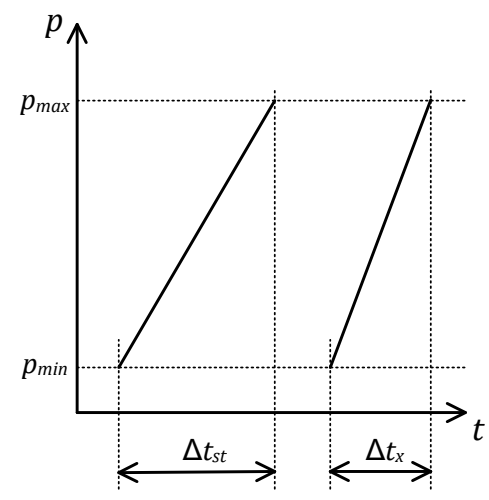

Figure 2: Successive pressure recordings in $V_{s t}$ and $V_{x}$.

One can see from equation (2) that $V_{x}$ is determined from the value of $V_{s t}$ (which can be calibrated with the gravimetric method) and from time interval measurements $\Delta t_{s t}$ and $\Delta t_{x}$. This means that only the repeatability of the pressure gauge can influence measurements, whether the gauge is calibrated or not. However, when the method is put into practice, it is much faster to record the pressure without caring about thresholds and to determine the pressure rise rate over time $\dot{p}$ afterwards, using a least squares method. Equation (1) can be then rewritten as:

$q(T)=V \dot{p}$,

and finally, from equation (3), the unknown volume is related to pressure rise rates via:

$V_{x}=V_{s t-x} \frac{\dot{p}_{s t-x}}{\dot{p}_{x}}$

\subsection{Volumes connected in series}

Similarly to 2.1, equations can be established for two volumes $V_{d}$ and $V_{s t-d}$ connected in series, where $V_{d}$ corresponds to the dead volume and $V_{s t-d}$ a standard volume. The calibration set-up for determining $V_{d}$ is shown Figure 3 .

The gas flows first in the volume composed of $V_{d}$ and $V_{s t-d}$ (phase 1 ), then the valve $v_{V_{s t-d}}$ is closed and the gas flow in $V_{d}$ only. Corresponding pressure rise rates $\dot{p}_{s t-d}$ and $\dot{p}_{d}$ are determined and $V_{d}$ can be expressed as:

$V_{d}=V_{s t-d} \frac{\dot{p}_{s t-d}}{\dot{p}_{d}-\dot{p}_{s t-d}}$.

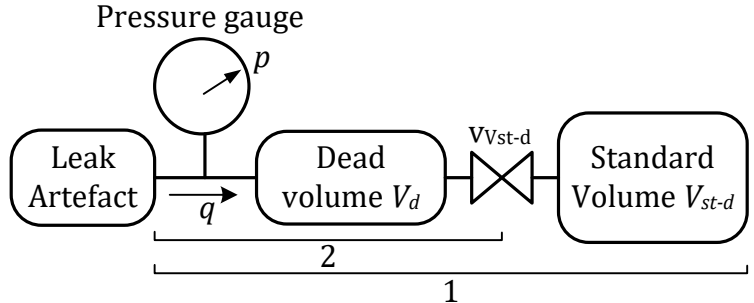

Figure 3: Calibration set-up, volumes connected in series.

\subsection{Calibration process equations}

Introducing the ratios $K_{d}$ and $K_{x}$ written as:

$K_{d}=\frac{\dot{p}_{s t-d}}{\dot{p}_{d}-\dot{p}_{s t-d}}\left(\equiv \frac{V_{d}}{V_{s t-d}}\right)$

and:

$K_{x}=\frac{\dot{p}_{s t}}{\dot{p}_{x}}\left(\equiv \frac{V_{x}+V_{d}}{V_{s t-x}+V_{d}}\right)$,

leads to expressions for $V_{d}$ and $V_{x}$ as follows:

$V_{d}=V_{s t-d} K_{d}$,

and:

$V_{x}=K_{x} V_{s t-x}+\left(K_{x}-1\right) V_{d}$.

Presentation of the measured quantities in a ratio form highlights the fact that the pressure gauge correction can be cancelled if the pressure response is linear or has been linearised previously. Ratio determinations are thus independent of the pressure gauge drift over time.

From equation (9), one can see that when $K_{x}$ tends towards unity (in other words when the values of $V_{x}$ and $V_{s t}$ lie close to each other), the contribution of the uncertainty of $V_{d}$ tends towards zero.

\section{INFLUENCE PARAMETERS}

Assuming the gas is perfect in the working pressure range, the calibration method relies mainly on the stability of the gas flow rate and a constant and identical gas temperature in the different volumes, during pressure rise rate measurements. Degassing of internal walls of the volumes is supposed to be negligible.

\subsection{Temperature}

Replacing the throughput (equation (1)) by the molar flow rate $q_{v}$ [4], we obtain for a constant gas temperature $T$ :

$q_{v}=V \frac{\Delta p}{\Delta t} \frac{1}{R T}$

where $R$ is the molar gas constant. Let us suppose that $T_{x}$ and $T_{s t-x}$ are gas temperatures during measurements of $\dot{p}_{x}$ and $\dot{p}_{s t-x}$ respectively. If the temperature difference is:

$\Delta T_{x}=T_{x}-T_{s t-x}$ 
the relative difference $\varepsilon_{x}$ is:

$\varepsilon_{x}=\frac{\Delta T_{x}}{T_{x}}$.

If $\varepsilon_{x} \ll 1$, the calculation leads to a ratio $K_{x}\left(\Delta T_{x}\right)$ which takes into account the temperature difference:

$K_{x}\left(\Delta T_{x}\right)=K_{x}\left(1+\varepsilon_{x}\right)$.

A similar calculation can be performed with the dead volume measurement. Indices " $d$ " and "st $d$ " are used instead of " $x$ " and "st $-x$ ". We then have:

$\Delta T_{d}=T_{d}-T_{s t-d}$,

and:

$\varepsilon_{d}=\frac{\Delta T_{d}}{T_{s t-d}}$.

Assuming $\varepsilon_{d} \ll 1$, the ratio $K_{d}\left(\Delta T_{d}\right)$ which finally takes into account the temperature difference is:

$K_{d}\left(\Delta T_{d}\right)=K_{d} \frac{1+\varepsilon_{d}}{1-K_{d} \times \varepsilon_{d}}$.

The other issue when measuring the pressure rise rate $\Delta p / \Delta t$ in a closed volume is the temperature variation rate $\Delta T / \Delta t$. A thermal perturbation term $(\Delta p / \Delta t)_{t h}$ arises which can be linked to $\Delta T / \Delta t$ by deriving the perfect gas law:

$\left(\frac{\Delta p}{\Delta t}\right)_{t h}=\frac{p}{T} \frac{\Delta T}{\Delta t}$.

This perturbation can be experimentally quantified if the flow rate $q$ is isolated from the pressure gauge and the volumes (during $V_{d}$ and $V_{x}$ determinations) so that the residual pressure rate can be measured.

\subsection{Flow rate stability}

In this work, a metal capillary leak was used to generate the gas flow rate. The downstream pressure of the capillary $p_{d}$ is the working pressure in the flowmeter. The upstream pressure $p_{u}$ is controlled by means of a pressure controller. The leak conductance $C$ depends on $p_{u}$ and the leak temperature $T_{l}$ via a temperature coefficient which absolute value is about $0.1-0.3 \% \cdot \mathrm{K}^{-1}$ [5]. The generated molar flow rate $q_{v}$ can be written as:

$q_{v}=C\left(p_{u}, T_{0}\right)\left(p_{u}-p_{d}\right) \alpha\left(T_{l}-T_{0}\right) \frac{1}{R T_{l}}$

where $T_{0}$ is an arbitrary reference temperature. For given temperature $T_{l}$ and controlled upstream pressure $p_{u}$, the flow rate depends on the downstream pressure stability. Assuming $p_{u m} \gg$ $p_{d m}$, we obtain:

$\frac{d q_{v}}{q_{v}} \cong \frac{-d p_{d}}{p_{u}}$.

This equation helps one choose the optimal leak conductance such that the flow rate stability is compatible with the targeted uncertainty.

\subsection{Ratio of pressure rise rates stability}

Measuring the pressure rise rates successively in the standard volume and the calibrated volume takes a few minutes during which the whole calibration system is likely to drift. In particular, the ambient temperature variation affects the flow rate stability (equation (18)). We illustrate in Figure 4 a variation of the pressure rise rate in the standard volume $V_{s t-x}$ as a function of time.

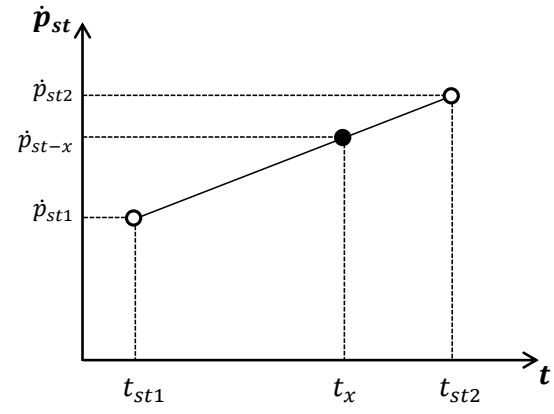

Figure 4: Interpolation of pressure rise rate measurements in the standard volume.

The pressure rise rate in the standard volume increased from $\dot{p}_{s t 1}$ to $\dot{p}_{s t 2}$ between $t_{s t 1}$ and $t_{s t 2}$. We evaluate what it should have been at the time $t_{x}$ when $\dot{p}_{x}$ was measured, by the simple interpolation formula:

$\dot{p}_{s t-x}=\dot{p}_{s t 1}+\left(\dot{p}_{s t 2}-\dot{p}_{s t 1}\right) \frac{t_{x}-t_{s t 1}}{t_{s t 2}-t_{s t 1}}$,

and use this value to determine the ratio $K_{x}$.

\section{EXPERIMENTAL SET-UP}

The volume calibration set-up is implemented with our constant-volume flowmeter facility shown Figure 5.

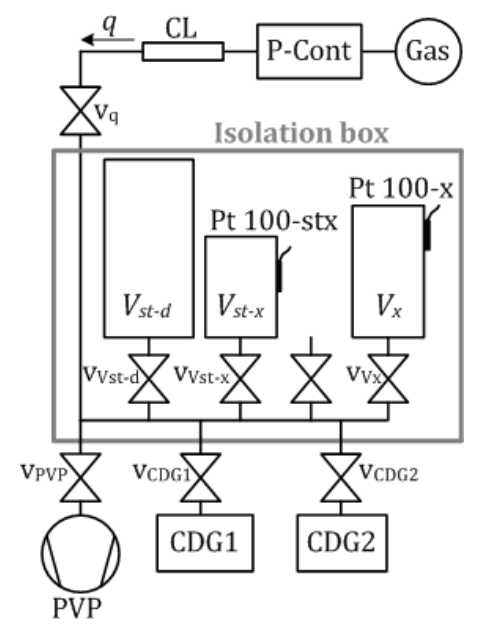

Figure 5: Experimental set-up using the constant-volume flowmeter. CDG1: Capacitance diaphragm gauge 690A, $100 \mathrm{~Pa}$ full range (MKS Instruments, United states); CDG2: capacitance diaphragm gauge $220 \mathrm{~A}, 10 \mathrm{kPa}$ full range (MKS Instruments); CL: Capillary leak (Inficon, Germany); P-Cont: Pressure controller DPI515 (Druck, Germany); PVP: Primary vacuum pump (vane pump); $\mathrm{v}_{\mathrm{xx}}$ : Bellows valves (Flowlink, France); Pt100-x and Pt100-stx: Pt100 thermometers. 
This flowmeter determines flow rates from the pressure rise rate measurement in a known volume [2]. It is composed of volumes of different capacities, two capacitance diaphragm gauges (CDG) connected in parallel to the tube leading the gas flow rate. A suitable combination of the standard volume and CDG is used upon the flow $q_{v}$ to be calibrated. Volumes and tubing are placed in an insulated box $\left(63 \times 42 \times 23 \mathrm{~cm}^{3}\right)$ to ensure better temperature stability.

\section{PROCEDURE AND UNCERTAINTY}

Preliminary observations with different configurations of volume sizes allowed defining a suitable procedure and guiding the uncertainty analysis.

\subsection{Preliminary measurements}

For leak calibrations, the pressure is usually recorded for one minute with a rate of one measurement per second. The pressure rise rate is then determined by means of a linear least squares modelling. For volume calibrations, one needs to close and open valves and also pump down the volumes, for different purposes: to switch from one volume to another, to set the pressure to a value compatible with the pressure gauge range or to proceed with a residual pressure rate recording. These operations create pressure jumps and consequently temperature disturbances which take some time to stabilise. The induced transition phase is shown Figure 6 for a pressure rise rate measured in a total volume (dead volume and standard volume) of about $590 \mathrm{~cm}^{3}$. The stabilisation in this example was reached in about four minutes. However, this stabilisation phase has a variable duration which depends for instance on the volume size or the pressure jump magnitude. During a calibration, this phase should be mastered to achieve the targeted uncertainty.

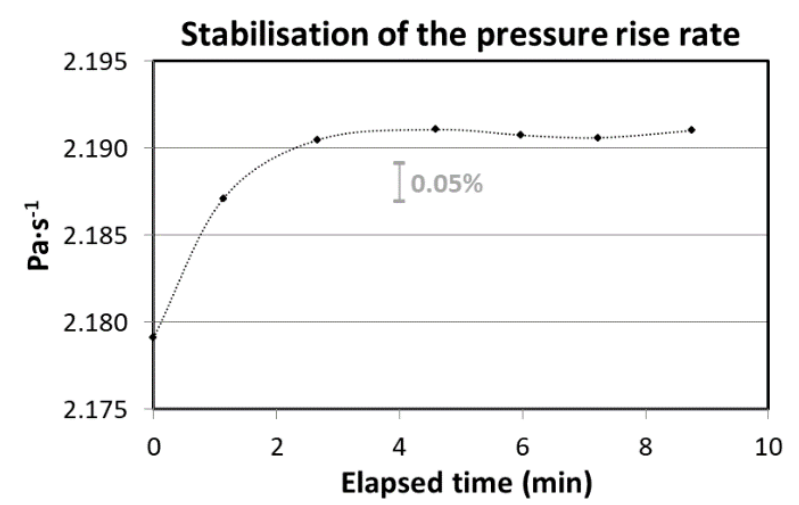

Figure 6: Transition phase of the measured pressure rise rate after switching from one volume to another.

The relative experimental standard deviation of the modelled pressure rise rate is about $8 \cdot 10^{-5}$ for these measurements.

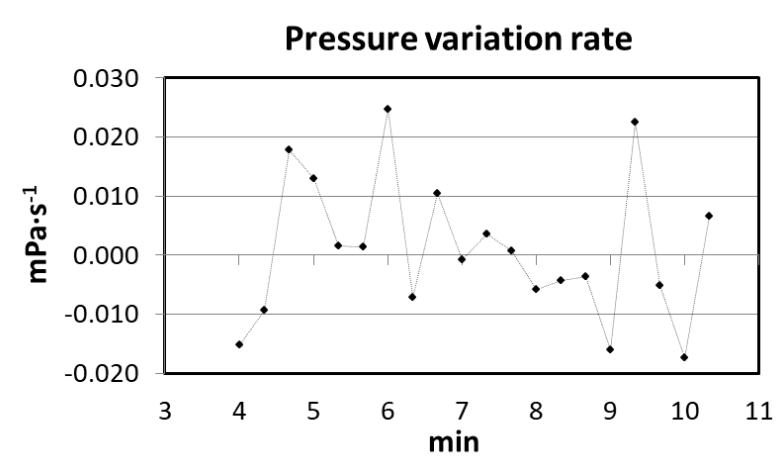

Figure 7: Pressure variation rate determined from the recording of pressure without flow.

Residual pressure rise rate measurements were performed, over an hour or more, in well-sealed 304L stainless steel volumes to evaluate the range of temperature variation rate (degassing and absorption being assumed negligible) from equation (17) and its contribution on the uncertainty.

The graph of Figure 7 shows the residual pressure variation rate $\dot{p}_{s t-r e s}$ determined from the recording of the pressure over time in a closed total volume of $360 \mathrm{~cm}^{3}$. One can see that $\dot{p}_{s t-r e s}$ changes sign in a short time corresponding to a measurement sequence. The mean slope $\overline{\dot{p}_{\text {st-res }}}$ over an hour is estimated to be $5.5 \times 10^{-7} \mathrm{~Pa} \cdot \mathrm{m}^{3} \cdot \mathrm{s}^{-1}$, which corresponds to a temperature variation rate of $0.25 \mathrm{mK} \cdot \mathrm{min}^{-1}$ (equation (17)). We generally obtain an agreement of better than $15 \%$ between temperature rates estimated from residual pressure rates and those estimated with Pt100 sensors. Typical absolute temperature rates in the isolation box placed in our air-conditioned room are below $1.0 \mathrm{mK} \cdot \mathrm{min}^{-1}$.

\subsection{Procedure}

Preliminary observations gave guidance to determine the ratio $K_{x}$ (or $K_{d}$ ). A sequence of three measurements of $\dot{p}_{x}$ (or $\dot{p}_{d}$ ) is performed between two sequences of three measurements of $\dot{p}_{s t-x}$ (or $\dot{p}_{s t-d}$ ) in order to apply equation (20). The pressure rise rate is obtained from the pressure recording over one minute (see $\S 5.1$ ). A stabilisation time (see Figure 6) before each measurement sequence of $\dot{p}$ is respected such that the relative difference between two successive determined values of $\dot{p}$ lies below $0.02 \%$. An alternation of four sequences of $\dot{p}_{s t-x}\left(\right.$ or $\left.\dot{p}_{s t-d}\right)$ determinations and three sequences of $\dot{p}_{x}$ (or $\dot{p}_{d}$ ) determinations allow us to obtain three determinations of $K_{x}$ (or $K_{d}$ ) and thus three values of $V_{x}$ (or $\left.V_{d}\right)$.

A measurement of the residual pressure rate is performed in each volume to check whether it lies in the typical range expected given the maximum temperature rate of $1.0 \mathrm{mK} \cdot \mathrm{min}^{-1}$ (see equation (17)). Rates are determined from pressure recording of at least two minutes. A residual pressure rate $\dot{p}_{x-r e s}$ in the calibrated volume $V_{x}$ significantly 
above the typical observed values would indicate some degassing (or absorption) of the volume walls which then should be subtracted from the determined values of $\dot{p}_{x}$.

\subsection{Uncertainty budget}

The uncertainty budget is drawn using data from the calibration of a $100 \mathrm{~cm}^{3}$ volume identified as V100 (used for the comparison with the gravimetric volume calibration method presented §6). Experimental data are found in Table 1.

Uncertainties in $V_{d}$ and $V_{x}$ are calculated using the variances propagation law from equations (8) and (9), including the repeatabilities estimated with the experimental deviations $s\left(V_{d}\right)$ and $s\left(V_{x}\right)$ :

$\frac{u\left(V_{d}\right)}{V_{d}}=\sqrt{\left[\frac{s\left(V_{d}\right)}{V_{d}}\right]^{2}+\left[\frac{u\left[K_{d}\left(\Delta T_{d}\right)\right]}{K_{d}}\right]^{2}+\left[\frac{u\left(V_{s t-d}\right)}{V_{s t-d}}\right]^{2}}$,

and:

$$
u\left(V_{x}\right)=\sqrt{\begin{array}{c}
{\left[s\left(V_{x}\right)\right]^{2}+\left[K_{x}\left(\Delta T_{x}\right) u\left(V_{s t-x}\right)\right]^{2}+} \\
{\left[\left(K_{x}\left(\Delta T_{x}\right)-1\right) u\left(V_{d}\right)\right]^{2}+} \\
{\left[\left(V_{s t-x}+V_{d}\right) u\left(K_{x}\left(\Delta T_{x}\right)\right)\right]^{2}}
\end{array}}
$$

Table 1: Quantities measured or used for the calibration of a $100 \mathrm{~cm}^{3}$ volume.

\begin{tabular}{|c|l|}
\hline Quantity & Value \\
\hline$q_{v}$ & $\begin{array}{l}4,9 \times 10^{-9} \mathrm{~mol} \cdot \mathrm{s}^{-1} \\
\left(1,2 \times 10^{-5} \mathrm{~Pa} \cdot \mathrm{m}^{3} \cdot \mathrm{s}^{-1} \mathrm{à} 20^{\circ} \mathrm{C}\right)\end{array}$ \\
\hline$p_{u}$ & $700 \mathrm{kPa}$ \\
\hline$p_{d}$ & $36 \mathrm{~Pa}<p_{d}<44 \mathrm{~Pa}$ \\
\hline$V_{d}$ & $59.9 \mathrm{~cm}^{3}$ \\
\hline$V_{s t-d}\left(u_{V_{s t-d}}\right)$ & $296.991(0.076) \mathrm{cm}^{3}$ \\
\hline$V_{x}$ & $100.1 \mathrm{~cm}^{3}$ \\
\hline$V_{s t-x}\left(u_{V_{s t-x}}\right)$ & $76.103(0.019) \mathrm{cm}^{3}$ \\
\hline$K_{d}$ & 0.201 \\
\hline$K_{x}$ & 1.18 \\
\hline$\dot{p}_{d}$ & $0.19 \mathrm{~Pa} \cdot \mathrm{s}^{-1}$ \\
\hline$\dot{p}_{s t-d}$ & $0.032 \mathrm{~Pa} \cdot \mathrm{s}^{-1}$ \\
\hline$\dot{p}_{x}$ & $0.072 \mathrm{~Pa} \cdot \mathrm{s}^{-1}$ \\
\hline$\dot{p}_{s t-x}$ & $0.085 \mathrm{~Pa} \cdot \mathrm{s}^{-1}$ \\
\hline$T$ & $20.5{ }^{\circ} \mathrm{C}$ \\
\hline$\Delta T / \Delta t$ & $0.19 \mathrm{mK} \cdot \mathrm{min}^{-1}$ \\
\hline$\Delta T_{x}$ & $-0.01 \mathrm{~K}$ \\
\hline$s\left(V_{d}\right)$ & $0.031 \mathrm{~cm}$ \\
\hline$s\left(V_{x}\right)$ & $0.021 \mathrm{~cm}$ \\
\hline
\end{tabular}

Thus, first we have to calculate the ratios $K_{d}$ and $K_{x}$ uncertainties from equations (6), (7), (13) and (16). Prior to this calculation, we recall the following statements: the method used for the calibration cancels the error of the gauge so there is no systematic uncertainty component for the ratios; the temperature variation rate induces in the short term a residual pressure rate which can be regarded as a noise (see Figure 7) on the pressure rise rate measured in the presence of $q_{v}$; the relative variation of $q_{v}$ due to $p_{d}$ variation (Table 1 ) is at the maximum $1.1 \times 10^{-5}$ (equation (19)) and can be neglected; the procedure described in $\S 3.3$ allows to contain drifts of the whole calibration system. Moreover, the experimental standard deviations respectively of the ratios $s\left(K_{x}\right)\left(\right.$ or $s\left(K_{d}\right)$ ) and the volumes $s\left(V_{x}\right)$ (or $s\left(V_{d}\right)$ ) are fully correlated. Finally, the remaining uncertainty components of the ratios are those related to temperature differences $\Delta T_{x}$ and $\Delta T_{d}$ (see $\S 3$ ). $\Delta T_{x}$ is measured with two $\mathrm{Pt} 100$ sensors placed on the volume walls. $\Delta T_{d}$ is too hard to measure since the dead volume is somewhat spatially extended. Consequently, it was decided to consider $\Delta T_{x}$ and $\Delta T_{d}$ as non-applied corrections with $\Delta T_{d}$ arbitrary fixed at $0.1 \mathrm{~K}$ in the experimental conditions and $\Delta T_{x}$ as it is estimated with a Pt100, with a minimum value of $0.02 \mathrm{~K}$. The variance propagation law thus gives [6] for uncertainties $u\left[K_{d}\left(\Delta T_{d}\right)\right]$ and $u\left[K_{x}\left(\Delta T_{x}\right)\right]$ :

$\frac{u\left[K_{d}\left(\Delta T_{d}\right)\right]}{K_{d}}=\left(1+\frac{V_{d}}{V_{s t-d}}\right) \frac{s\left(K_{d}\right)}{K_{d}}+\frac{1}{2}\left|1-\frac{1+\varepsilon_{d}}{1-K_{d} \times \varepsilon_{d}}\right|$,

and:

$\frac{u\left[K_{x}\left(\Delta T_{x}\right)\right]}{K_{x}}=\frac{s\left(K_{x}\right)}{K_{x}}+\frac{1}{2}\left|1+\varepsilon_{x}\right|$.

Even if $s\left(K_{d}\right)$ is neglected, one should pay attention to the choice of $V_{s t-d}$ value regarding the sensitivity coefficient in equation (23).

Standard volumes (sample cylinders of $304 \mathrm{~L}$ stainless steel) are connected to the input port of a bellows valve VL214 from Flowlink (France) which has a dead volume of about $0.681 \mathrm{~cm}^{3}$. Uncertainties of the standard volumes given in Table 1 include the calibration uncertainty of the gravimetric method and that of the dead volume of the bellow valve $u_{b e l}$. The latter was estimated to be $0.012 \mathrm{~cm}^{3}$ from a study of the manufacturer drawings and several calibrations with the gravimetric method performed on valves of the same type randomly selected. As the volume V100 was also connected to a bellows valve of the flowmeter to be calibrated, $u_{b e l}$ was quadratically summed with $u\left(V_{x}\right)$ of equation (22). We eventually estimated $u\left(V_{100}\right)=0.032 \mathrm{~cm}^{3}(0.031 \%$ in relative value).

\section{COMPARISON WITH THE GRAVIMETRIC METHOD}

A series of six volume artefacts of $1.5 \mathrm{~cm}^{3}$, $10 \mathrm{~cm}^{3}, 100 \mathrm{~cm}^{3}, 300 \mathrm{~cm}^{3}, 500 \mathrm{~cm}^{3}$ and $2500 \mathrm{~cm}^{3}$ were calibrated to characterise the comparison method with a transfer leak flow rate, in the range 1 to $3000 \mathrm{~cm}^{3}$. The reference value is given by the gravimetric calibration method. Table 2 summarises the comparison results: $V_{\text {nom }}$ is the nominal value of the calibrated volume, $d$ is the difference between 
$V_{q}$ determined with the studied calibration method and $V_{g}$ determined with the gravimetric method:

$d=V_{q}-V_{g}$.

$u\left(V_{q}\right)$ is the standard uncertainty of $V_{q}$ and $E_{n}$ is the normalised error

$$
E_{n}=\frac{d}{\sqrt{\left(2 u\left(V_{q}\right)\right)^{2}+\left(2 u\left(V_{g}\right)\right)^{2}}} .
$$

Table 2: Comparison results.

\begin{tabular}{|l|c|c|r|}
\hline $\begin{array}{c}V_{\text {nom }}\left(V_{\text {st-x }}\right) \\
\mathrm{cm}^{3}\end{array}$ & $\begin{array}{c}d \\
\mathrm{~cm}^{3}\end{array}$ & $\begin{array}{c}u\left(V_{q}\right)\left(u\left(V_{q}\right) / V_{q}\right) \\
\mathrm{cm}^{3}\end{array}$ & $E_{n}$ \\
\hline $1.5(25)$ & 0.032 & $0.020(1.3 \%)$ & 0.77 \\
\hline $10(25)$ & 0.015 & $0.024(0.23 \%)$ & 0.28 \\
\hline $100(75)$ & -0.006 & $0.032(0.032 \%)$ & -0.08 \\
\hline $300(500)$ & -0.010 & $0.096(0.032 \%)$ & -0.04 \\
\hline $500(300)$ & 0.004 & $0.15(0.030 \%)$ & 0.01 \\
\hline $2500(500)$ & 1.3 & $1.0(0.041 \%)$ & 0.66 \\
\hline
\end{tabular}

The nominal value $V_{s t-x}$, amongst the available standard volume, which was used for the calibration, is indicated together with $V_{\text {nom }}$. The volumes of 300 and $500 \mathrm{~cm}^{3}$ are actually standard volumes which were cross compared.

Comparison results are satisfactory for all the calibrated volumes with an absolute value of the normalised error less than one. The higher standard uncertainty is for the smaller volumes. It is estimated to be about $2 \%$ for a $1 \mathrm{~cm}^{3}$ volume.

\section{SUMMARY}

A volume calibration method implemented using the LCM-LNE-Cnam constant-volume flowmeter has been characterised. Successive measurements of pressure rise rates, due to a steady leak rate, are performed successively in a standard volume and in an unknown volume. From the value of the standard volume and the ratio of pressure rise rates, the unknown volume can be determined. Special care taken in measurement and data analysis has led to a standard relative uncertainty between $0.03 \%$ and $2 \%$ for volumes ranging from 1 to $3000 \mathrm{~cm} 3$, roughly an order of magnitude higher than capabilities of the gravimetric volume calibration.

\section{REFERENCES}

[1] EURAMET e.V., Technical Committee for Flow, Guidelines on the determination of uncertainty in gravimetric volume calibration, Calibration guide cg-19, Version 2.1, 03/2012.

[2] P. Otal, F. Boineau, J.-C. Legras, Etalonnage des fuites d'hélium: méthodes de référence et dissémination de $10^{-10} \mathrm{~Pa} \cdot \mathrm{m}^{3} \cdot \mathrm{s}^{-1}$ à $10^{-2} \mathrm{~Pa} \cdot \mathrm{m}^{3} \cdot \mathrm{s}^{-1}$, Revue française de métrologie, vol. 1, n ${ }^{\circ} 17$, pp. 39, 2009 (in French).

[3] J. Setina, B. Erjavec, Volume determination of a vacuum vessel by pressure rise method, XIX IMEKO World Congress, September 6-11, 2009, Lisbon, Portugal.

[4] K. Jousten et al., Final report of key comparison CCM.P-K12 for very low helium flow rates (leak rates), Metrologia, vol. 50, no 1A, p. 07001, 2013, DOI:

https://doi.org/10.1088/0026-1394/50/1A/07001

[5] D. Mari et al., «Realization, characterization and measurements of standard leak artefacts », Measurement, vol. 61, p. 249-256, 2015, doi: https://doi.org/10.1016/j.measurement.2014.10.045

[6] AFNOR, "Evaluation of measurement data-Guide to the expression of uncertainty in measurement", JCGM 100, Paris, AFNOR, 2008, 121 p. 